\title{
Synthesis of Hierarchical TS-1 and Its Recycling Catalytic Property for Oxidative Desulfurization
}

\author{
Jinglong Huai ${ }^{1}$, Xiaoxue Liu, ${ }^{2,}$, Yinhai Zhang ${ }^{1}$, Yang Yang ${ }^{1}$, Yan Gou ${ }^{1}$, Li Qin ${ }^{1}$, Jinling Shan ${ }^{1}$, \\ Yancheng Zheng ${ }^{1}$ and Hao $\mathrm{Li}^{1{ }^{1 *}}$ \\ ${ }^{1}$ College of Chemistry and Environmental Engineering, Yangtze University, Jingzhou, 434023, China \\ ${ }^{2}$ College of Agriculture, Yangtze University, Jingzhou, 434000, China \\ ${ }^{*}$ Corresponding Authors: Xiaoxue Liu. Email: xiaoxueliu@yangtzeu.edu.cn; Hao Li. Email: haoli@yangtzeu.edu.cn \\ Received: 28 July 2021 Accepted: 27 September 2021
}

\begin{abstract}
Because of its unique pore structure, good hydrothermal stability and high specific surface area, hierarchical TS-1 zeolite (HTS-1) has become an important catalyst for the deep oxidative desulfurization of fuel oils. In this work, HTS-1 has been successfully synthesized by a hydrothermal crystallization method using the $\mathrm{C}-\mathrm{SiO}_{2}$ composite as both silicon source and mesoporous template, tetrapropylammonium hydroxide as microporous template, and tetrabutylorthotitanate as titanium source. The $\mathrm{C}-\mathrm{SiO}_{2}$ composite is obtained by mild carbonization of the $\mathrm{SiO}_{2} / \mathrm{T}-40$ (Tween 40 ) xerogel, which is prepared by the two step sol-gel method. The reaction conditions for the oxidative desulfurization (ODS) of dibenzothiophene (DBT) and 4,6-dimethyldibenzothiophene (4,6-DMDBT) over HTS-1 are optimized systematically, and the recycling performances of HTS-1 are investigated in detail. After the 15th run, HTS-1 still maintains high DBT conversion (90.6\%) and 4,6-DMDBT conversion (86.0\%) without deactivation. The samples before and after recycle tests are characterized by XRD, FT-IR, $\mathrm{CHN}$ analysis, UV-Vis and SEM techniques. The results indicate that the crystal structure and morphology of regenerated HTS-1 samples are well kept, which accounts for the good structural stability and reusability of HTS-1. In addition, active intermediates for the ODS of bulky organic sulfides over HTS-1, i.e., Ti-peroxo $\left(\mathrm{Ti}-\mathrm{OO}^{\mathrm{t}} \mathrm{Bu}\right.$ ) species, are captured by the UV-Vis technique. Finally, a possible reaction mechanism for the ODS process over HTS-1 is proposed.
\end{abstract}

\section{KEYWORDS}

Hierarchical TS-1; oxidative desulfurization; recycling property; Ti-peroxo species

\section{Introduction}

The combustion of sulfur-containing fuel oils leads to the emission of $\mathrm{SO}_{x}$ gases, which causes harm to environment and human health, such as acid rain and lung cancer. Thus, many countries have established strict regulations to limit the sulfur content in fuel oils to less than $10 \mathrm{ppm}$. Industrially, hydrodesulfurization (HDS) technology is usually used to remove organic sulfur components in fuel oils. Sulfur element is converted into $\mathrm{H}_{2} \mathrm{~S}$ gas for discharge, and sulfur-containing compounds are converted into aliphatic hydrocarbons [1,2]. However, the HDS technology usually requires high temperature (573$723 \mathrm{~K}$ ) and pressure $\left(3.0-5.0 \mathrm{MPa}\right.$ of $\left.\mathrm{H}_{2}\right)$, and it is difficult to remove refractory sulfur compounds such 
as thiophene, benzothiophene and their derivatives [3]. Therefore, some non-hydrodesulfurization methods, such as adsorption desulfurization [4], extraction desulfurization [5], biodesulfurization [6] and oxidative desulfurization (ODS) [7], have been developed to overcome the drawbacks. Among these technologies, the ODS has attracted much attention because it can efficiently remove thiophenic compounds under mild conditions.

The ODS was proposed in the 1990s [8], it generally consists of two processes: the first step is oxidation of organic sulfur compounds in fuel oils, and the following step is removal of oxidation products from the treated fuel oils by extraction or adsorption [9]. The early catalysts used for ODS process are formic acid [7], sulfuric acid [9] and acetic acid [10]. Later, various types of catalysts for ODS of liquid fuels have been reported, such as polyoxometalates and their composite materials [11,12], supported metal oxides [13], and titanium-containing molecular sieves [14-21]. Among these catalysts, titanium-containing molecular sieves have become a research hotspot in recent years due to their excellent performances for the ODS. Titanium silicalite (TS-1) has a good removal effect on small molecular sulfides. However, due to its intrinsic micropores (about $0.55 \mathrm{~nm}$ ) which limit the diffusion of bulky reactants to active sites, it has little effect on the ODS of bulky sulfur compounds [15]. Titanium-containing mesoporous molecular sieves $[16,22,23]$ have larger pore sizes, which can effectively enhance the mass transfer of bulky sulfur compounds. Unfortunately, because of the amorphous nature of pore walls, their hydrothermal stability is poor. Hierarchical TS-1 molecular sieve (HTS-1) is a kind of TS-1 zeolite with both micropores and mesopores or macropores. It not only overcomes the diffusion limitation of bulky reactants, but also has good hydrothermal stability, so it has aroused growing interest in the past few years.

Generally, the preparation methods of HTS-1 can be divided into top-down and bottom-up approaches according to the strategies for introducing mesopores. The top-down approach is to introduce additional mesopores into the TS-1 through post-treatments, mainly including alkali [24-27] or acid treatment [17]. The bottom-up approach usually refers to the addition of soft or hard templates that can produce a mesopore structure during the crystallization of TS-1, and the as-synthesized TS- 1 powders are calcined to remove the template to obtain HTS-1. Soft templates usually include surfactants [16], cationic polymers $[19,28]$ and organosilane agents [29]. The main disadvantage of soft templates is that a large amount of expensive organic materials must be sacrificed. Hard templates mainly include carbon black [20], carbon nanotubes [30] and mesoporous carbon CMKs [21]. Soft templates, such as amphiphilic organosilanes, can form covalent bonds with inorganic materials (for example, $\mathrm{SiO}_{2}$ and/or $\mathrm{TiO}_{2}$ ) to enhance their interaction with growing zeolite domains, avoiding the 'phase separation' phenomenon. On the contrary, the interaction force between common hard templates and growing zeolite domains is weak. During the hydrothermal synthesis process, the hard templates may be excluded from TS-1 crystals due to the 'phase separation' phenomenon, so it is difficult to form a good intracrystalline mesoporous structure.

To solve this problem, Du et al. [31] have used polyvinyl alcohol modified multi-wall carbon nanotubes (MWCNTs) as the template to prepare HTS-1 by the steam-assisted crystallization. Polyvinyl alcohol with abundant $-\mathrm{OH}$ groups can connect MWCNTs and TS-1 precursors, and the interaction between them is improved. Unfortunately, the mesopore volume of HTS-1 has not increased much compared with TS- 1. Wang et al. [20] have prepared HTS-1 by hydrothermal crystallization of $\mathrm{C}-\mathrm{SiO}_{2}-\mathrm{TiO}_{2}$ composites, which are prepared by carbonizing sucrose directly onto $\mathrm{SiO}_{2}$ and $\mathrm{TiO}_{2}$. The direct high-temperature carbonization of sucrose improves the interaction between carbon template and silica source, which is beneficial to the encapsulation of carbon template in the crystallization process of zeolite. However, since sucrose and inorganic species are only physically mixed and dispersed, the improvement may be poor. Our team [32] has prepared a uniformly hybrid xerogel by the sol-gel method using Tween 40 (T-40) as the mesoporous carbon source. By mild carbonization of hybrid xerogels, the $\mathrm{C}-\mathrm{SiO}_{2}-\mathrm{TiO}_{2}$ composite with high carbon content is obtained. The $\mathrm{SiO}_{2}$ and $\mathrm{TiO}_{2}$ particles are tightly encapsulated by carbon materials, indicating that the interaction between the carbon template and the inorganic species is 
improved. Thus, HTS-1 with high mesoporous volume is obtained by hydrothermal crystallization of the $\mathrm{C}-\mathrm{SiO}_{2}-\mathrm{TiO}_{2}$ composite, and it shows high catalytic activity and stability in the ODS of dibenzothiophene (DBT) and 4,6-dimethyldibenzothiophene (4,6-DMDBT).

In a previous communication [33], we have prepared $\mathrm{C}-\mathrm{SiO}_{2}$ composites by the similar method as reported in reference [32], and HTS-1 zeolites are synthesized by the hydrothermal crystallization of $\mathrm{C}-\mathrm{SiO}_{2}$ composites. Effects of the amount of T-40 on the catalytic performances of HTS- 1 for the ODS of DBT and 4,6-DMDBT are investigated, and the appropriate molar ratio of T-40/Si (0.14) is obtained. Here, the reaction conditions of the ODS of DBT and 4,6-DMDBT over HTS-1 are further optimized, the recycling catalytic performances of HTS-1 are investigated, and the catalytic mechanism of the ODS process is studied.

\section{Experimental}

\subsection{Materials}

Tetrabutylorthotitance (TBOT) and Tert-butyl hydroperoxide (TBHP, $~ 5.5 \mathrm{~mol} / \mathrm{L}$ in decane) were purchased from Sigma. Tetraethylorthosilicate (TEOS), Tween 40 (T-40), Dibenzothiophene (DBT), 4,6dimethyldibenzothiophene (4,6-DMDBT) and TBHP solution $\left(70 \%\right.$ in $\left.\mathrm{H}_{2} \mathrm{O}\right)$ were purchased from Aladdin. Tetrapropylammonnium hydroxide (TPAOH, $40 \mathrm{wt} . \%)$ was purchased from Alfa-Aesar. Isopropyl alcohol (IPA) and n-octane were purchased from Sinopharm Chemical Reagent Co., Ltd.

\subsection{Synthesis}

\subsubsection{Synthesis of $\mathrm{SiO}_{2} / \mathrm{T}-40$ Xerogel}

The $\mathrm{SiO}_{2} / \mathrm{T}-40$ xerogel was prepared following the two step sol-gel process [32,33]. Typically, $33.3 \mathrm{~g}$ of TEOS was mixed with $11.5 \mathrm{~g}$ of hydrochloric acid aqueous solution $(0.05 \mathrm{~mol} / \mathrm{L})$ at room temperature, and TEOS was hydrolyzed to obtain a clear solution. Also, $28.7 \mathrm{~g}$ of T-40 was dissolved in $60 \mathrm{~mL}$ deionized water at $313 \mathrm{~K}$. Then, the clear solution was added to the T-40 aqueous solution and kept stirring at $313 \mathrm{~K}$ for $2 \mathrm{~h}$. Then, a small amount of TPAOH was added dropwise, and a milky white gel was obtained. In the end, the gel was completely dried at $353 \mathrm{~K}$ and ground to obtain $\mathrm{SiO}_{2} / \mathrm{T}-40$ xerogel, which was named as ST. For comparison, $\mathrm{SiO}_{2}$ xerogel was synthesized in a similar way in the absence of T-40.

\subsubsection{Synthesis of $\mathrm{C}-\mathrm{SiO}_{2}$ Composite}

The $\mathrm{C}-\mathrm{SiO}_{2}$ composite was prepared by mild carbonization of ST powders [32,33]. Typically, ST powders $(10.0 \mathrm{~g})$ were dispersed in a dilute sulfuric acid solution $\left(\mathrm{H}_{2} \mathrm{O}: 100 \mathrm{~mL}, 98 \% \mathrm{H}_{2} \mathrm{SO}_{4}: 2.0 \mathrm{~mL}\right)$ under stirring at room temperature for $1 \mathrm{~h}$. The resulting slurry was completely dried in a drying oven $(373 \mathrm{~K})$, and then the temperature of oven was increased to $433 \mathrm{~K}$ and heat-treated at this temperature for around $12 \mathrm{~h}$. The obtained black solid was ground into powders, and the black powders were heated under a nitrogen flow up to $773 \mathrm{~K}$ for $12 \mathrm{~h}$ (heating rate: $1 \mathrm{~K} / \mathrm{min}$ ). The obtained $\mathrm{C}-\mathrm{SiO}_{2}$ composite was named as CS, and the carbon content of CS composite calcined in air at $823 \mathrm{~K}$ was $38.1 \%$.

\subsubsection{Synthesis of HTS-1}

HTS- 1 was synthesized according to the method reported in reference [33]. Typically, $0.31 \mathrm{~g}$ of TBOT was dissolved in $10 \mathrm{~mL}$ isopropyl alcohol, and the solution was placed in an ice water bath. To the stirred solution of TBOT, an aqueous solution of TPAOH $\left(6.9 \mathrm{~g}\right.$ of TPAOH and $4.6 \mathrm{~g}$ of deionized $\left.\mathrm{H}_{2} \mathrm{O}\right)$ was added dropwise. The solution was stirred at room temperature for $1 \mathrm{~h}$, and then heated to $343 \mathrm{~K}$ for about $2 \mathrm{~h}$ in order to evaporate the alcohol. After evaporating the alcohol, $2.9 \mathrm{~g}$ of CS powders were dispersed into the solution, and the mixture was stirred at room temperature for $4 \mathrm{~h}$. The molar composition of the resulting mixture was $1 \mathrm{SiO}_{2}: 0.03 \mathrm{TiO}_{2}: 0.45 \mathrm{TPAOH}: 16.20 \mathrm{H}_{2} \mathrm{O}$. Then, the mixture was under hydrothermal conditions at $443 \mathrm{~K}$ for $48 \mathrm{~h}$. Finally, the solid product was washed, dried and calcined at 
$823 \mathrm{~K}$ for $5 \mathrm{~h}$. Moreover, TS-1 was synthesized by hydrothermal crystallization of the $\mathrm{SiO}_{2}$ xerogel in a similar way.

\subsection{Treatment of Samples with TBHP Aqueous Solution}

In a typical treatment, $40 \mathrm{mg}$ of HTS- 1 was fed into a beaker, then $0.4 \mathrm{~mL}$ TBHP ( $70 \%$ in water) was added dropwise. The mixture was fully stirred and immersed using a glass rod. Finally, the mixture was ventilated and dried at room temperature for about $3 \mathrm{~h}$ to obtain a light yellow solid.

\subsection{Characterization}

The XRD patterns were recorded on a Panalytical Empyrea X-ray diffractometer with a $\mathrm{Cu}-\mathrm{K} \alpha$ radiation, and the scan angles $(2 \theta)$ were $5-50^{\circ}$. The UV-Vis spectra were measured with a PerkinElmer Lambda $650 \mathrm{~S}$ spectrometer, and the spectra were documented over 190-800 nm. The IR spectra were detected by a Nicolet 6700 infrared spectrometer with pure $\mathrm{KBr}$ as background. The measurements were carried out in the wavelength range of $400-4000 \mathrm{~cm}^{-1}$. The nitrogen adsorption-desorption isotherms were evaluated by a Micromeritics ASAP 2020HD88 physical adsorption instrument. Samples to be tested were first desorbed and activated in vacuum $(573 \mathrm{~K}, 4 \mathrm{~h})$, and then tested at a low temperature of $77 \mathrm{~K}$ (liquid nitrogen). The specific surface area $\left(\mathrm{S}_{\mathrm{BET}}\right)$ was evaluated using a BET equation. The pore size distribution and cumulative pore volume $\left(\mathrm{V}_{\text {meso }}\right)$ were calculated from the desorption branch of isotherm by the BJH model. The micropore volume $\left(\mathrm{V}_{\text {micro }}\right)$, external surface area $\left(\mathrm{S}_{\text {ext }}\right)$ and micropore area $\left(\mathrm{S}_{\text {micro }}\right)$ were calculated by the t-plot method. The carbon content of samples was analyzed by a Vario EL III elemental analyzer. The SEM and TEM images were taken by a TESCAN MIRA3 field emission scanning electron microscope and a JEOL JEM-2100F transmission electron microscope, respectively. The Ti content of samples was determined with a PerkinElmer Optima 8000 inductively coupled plasma emission spectrometer.

\subsection{Catalytic Activity}

DBT and 4,6-DMDBT were dissolved in n-octane to prepare model fuels with sulfur contents of 1000 and $500 \mathrm{ppm}$, respectively. Typically, DBT model fuel $(10.0 \mathrm{~mL})$ and catalyst $(50.0 \mathrm{mg})$ were fed into a $50 \mathrm{~mL}$ two-neck glass flask equipped with a condenser. The mixture was stirred and heated to $333 \mathrm{~K}$, then $120.0 \mu \mathrm{L}$ of TBHP was added to start the reaction. When the reaction reached the predetermined time, it was stopped. The reaction mixture was cooled and centrifuged to obtain oil phase and solid. The oil phase was analyzed on a GC 126N chromatography (FPD, HP-5 capillary column, $0.25 \mu \mathrm{m} \times 0.32 \mathrm{~mm} \times 30 \mathrm{~m}$ ). The residual DBT and 4,6-DMDBT were quantified by the method of peak area normalization. The conversions of DBT and 4,6-DMDBT $(X)$ are defined as the amount of sulfide reacted divided by the initial amount of sulfide added. In addition, the solid mixture was washed with hot acetonitrile $(323 \mathrm{~K})$ and centrifuged several times to obtain a supernatant. Then, the supernatant was concentrated in vacuum, and the residue was dried in a vacuum oven at room temperature for $24 \mathrm{~h}$ to give the oxidation product of DBT (or 4,6-DMDBT).

\subsection{Recycle Tests}

\subsubsection{Recycling Property of Samples Recovered by Centrifugation}

The cyclic experiments were proceeded in a $250 \mathrm{~mL}$ three-necked flask immersed in an oil bath and equipped with a condenser. The typical cyclic experiment procedure is similar to that described in Section 2.5, and the oxidation reactions are carried out according to the optimized conditions: the catalyst to oil ratio was $5.0 \mathrm{~g} / \mathrm{L}$, the molar ratio of TBHP/S was 3.0 , the reaction temperature was $333 \mathrm{~K}$, and the reaction time for DBT model fuel was $3 \mathrm{~h}$ (the reaction time for 4,6-DMDBT model fuel was $2 \mathrm{~h}$ ). The difference is that the dosage of catalyst for the first reaction was $750.0 \mathrm{mg}$. After the completion of the reaction, the reaction mixture was cooled and centrifuged to obtain oil phase and solid. The analysis 
of the oil phase is the same as described in Section 2.5. The solid was further centrifuged and washed with hot acetonitrile $(323 \mathrm{~K})$ for 5 times, and the amount of hot acetonitrile was $30 \mathrm{~mL}$ each time. Finally, the solid was dried at $383 \mathrm{~K}$ for $12 \mathrm{~h}$, and the obtained catalyst was denoted as HTS-1cx, where x represents the number of cyclic experiments. In each run, the dosage of TBHP and model fuels were calculated according to the ratio of catalyst to oil, the molar ratio of TBHP/S, and the mass of the catalyst obtained in the previous run.

\subsubsection{Recycling Property of Samples Regenerated by Calcination}

The typical cyclic experiments are similar to the procedure in Section 2.6.1. The only difference is that after centrifugation, hot acetonitrile washing and drying, the solid was calcined in air at $823 \mathrm{~K}$ for $5 \mathrm{~h}$ (heating rate: $1 \mathrm{~K} / \mathrm{min}$ ). The regenerated catalyst was denoted as HTS-1ccx, where $\mathrm{x}$ represents the number of cyclic experiments.

\section{Results and Discussion}

\subsection{Structural Characterizations}

As shown in Fig. 1, the XRD patterns of TS-1 and HTS-1 display diffraction peaks at $7.9^{\circ}, 8.8^{\circ}, 23.0^{\circ}$, $23.9^{\circ}$ and $24.3^{\circ}$, which belong to the characteristic peaks of MFI topology.

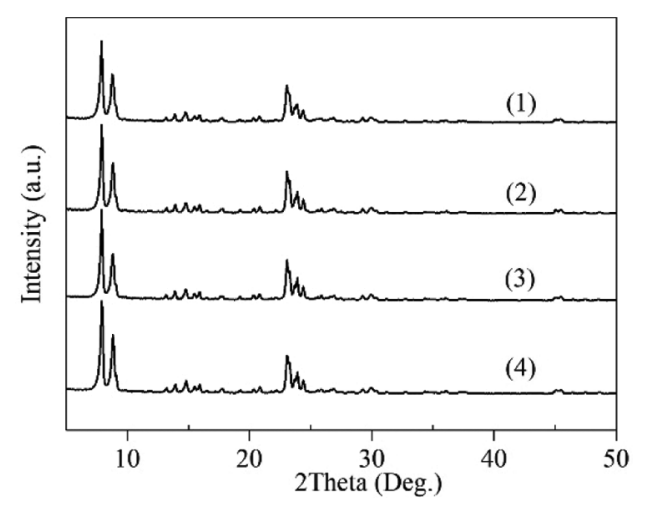

Figure 1: XRD patterns of TS-1 (1), HTS-1 (2), HTS-1cc15 regenerated from the ODS of DBT (3), and HTS-1cc15 regenerated from the ODS of 4,6-DMDBT (4)

Fig. 2a gives the UV-Vis spectra of TS-1 and HTS-1. Both of the two samples show an absorption band at $204 \mathrm{~nm}$, which is attributed to the isolated Ti (IV) species. An absorption band at $2330 \mathrm{~nm}$ is also detected for TS-1 and HTS-1, indicating the existence of some anatase $\mathrm{TiO}_{2}$ [34]. Moreover, a weak absorption band of HTS- 1 at $260-280 \mathrm{~nm}$ is observed, which is attributed to $\mathrm{Ti}^{{ }^{4+}}$ ions in an octahedral coordination with two water molecules in the coordination sphere or small hydrated oligomeric $\mathrm{TiO}_{x}$ species [35].

Fig. 3 shows the FT-IR spectra of TS-1 and HTS-1. The absorption peak at $960 \mathrm{~cm}^{-1}$ is attributed to the stretching vibration of the $\mathrm{Si}-\mathrm{O}-\mathrm{Ti}$ bond or $\mathrm{Si}-\mathrm{O}$ bond perturbed by framework Ti atoms [36]. In addition, the absorption peaks of the samples at $550 \mathrm{~cm}^{-1}$ and $1228 \mathrm{~cm}^{-1}$ are attributed to the characteristic of MFI topology [37].

The $\mathrm{N}_{2}$ adsorption-desorption isotherms and pore size distributions of TS- 1 and HTS-1 are shown in Fig. 4. TS-1 exhibits a typical type I isotherm, indicating that it has a microporous structure. HTS1 shows a typical IV-type isotherm with an obvious $\mathrm{H} 3$ hysteresis loop at $\mathrm{P} / \mathrm{P}_{0}>0.65$, revealing the presence of mesopores or macropores in the sample. The mesoporous pore size of TS-1 is widely distributed in 3-4 $\mathrm{nm}$ and 5-80 nm, while HTS-1 shows a clear mesopore/macropore distribution 
(4-85 nm) with the most probable pore size being $12.8 \mathrm{~nm}$ (Fig. 4b). Compared with TS-1, the $\mathrm{V}_{\text {micro }}$ of HTS-1 is similar, but the $\mathrm{S}_{\mathrm{BET}}, \mathrm{S}_{\mathrm{ext}}$ and $\mathrm{V}_{\text {meso }}$ of HTS-1 are significantly increased (Table 1).
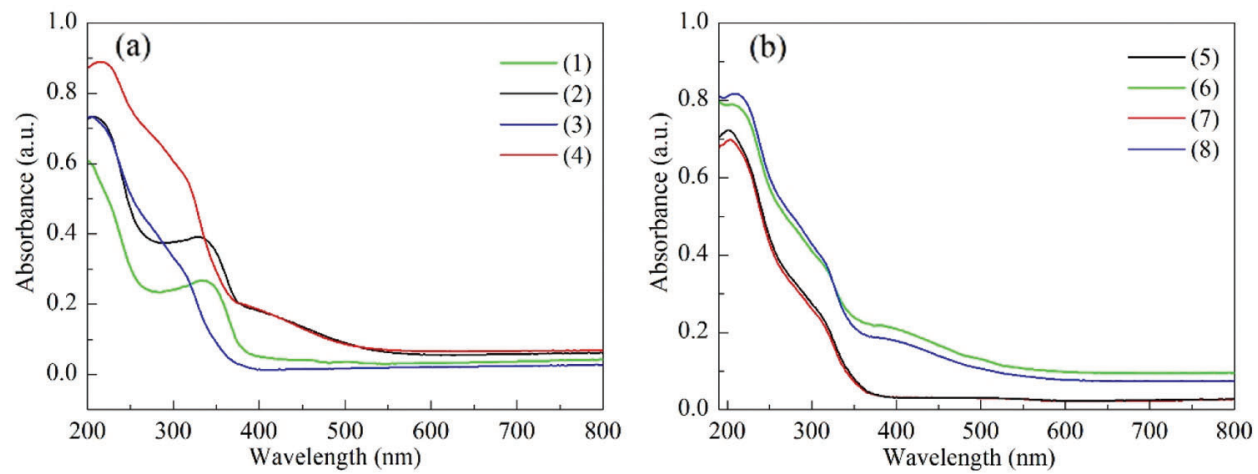

Figure 2: UV-Vis spectra of TS-1 and fresh HTS-1 samples (a), and regenerated HTS-1 samples (b): (1) TS-1, (2) TS-1 treated with TBHP, (3) fresh HTS-1, (4) fresh HTS-1 treated with TBHP, (5) HTS-1cc15 regenerated from the ODS of 4,6-DMDBT, (6) HTS-1cc15 regenerated from the ODS of 4,6DMDBT and treated with TBHP, (7) HTS-1cc15 regenerated from the ODS of DBT, (8) HTS$1 \mathrm{cc} 15$ regenerated from the ODS of DBT and treated with TBHP

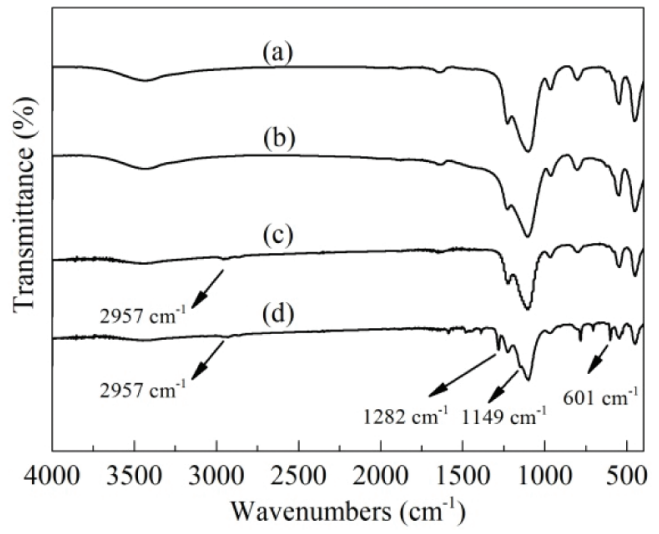

Figure 3: FT-IR spectra of TS-1, HTS-1 and HTS-1c5 samples: (a) TS-1, (b) HTS-1, (c) HTS-1c5 recovered from the ODS of DBT, (d) HTS-1c5 recovered from the ODS of 4,6-DMDBT
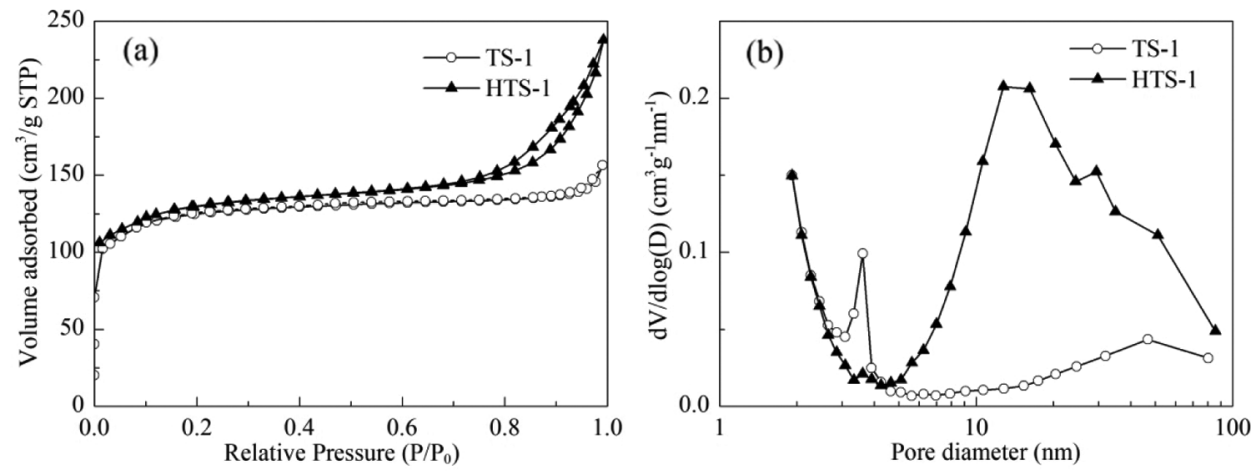

Figure 4: $\mathrm{N}_{2}$ adsorption-desorption isotherms (a) and pore size distributions (b) of TS-1 and HTS-1 
Table 1: Compositions and textural properties of TS-1 and HTS-1

\begin{tabular}{lllllll}
\hline Sample & $\mathrm{Si} / \mathrm{Ti}^{\mathrm{a}}$ & $\begin{array}{l}\mathrm{S}_{\mathrm{BET}} \\
\left(\mathrm{m}^{2} / \mathrm{g}\right)\end{array}$ & $\begin{array}{l}\mathrm{S}_{\text {micro }} \\
\left(\mathrm{m}^{2} / \mathrm{g}\right)\end{array}$ & $\begin{array}{l}\mathrm{S}_{\text {ext }} \\
\left(\mathrm{m}^{2} / \mathrm{g}\right)\end{array}$ & $\begin{array}{l}\mathrm{V}_{\text {micro }} \\
\left(\mathrm{cm}^{3} / \mathrm{g}\right)\end{array}$ & $\begin{array}{l}\mathrm{V}_{\text {meso }} \\
\left(\mathrm{cm}^{3} / \mathrm{g}\right)\end{array}$ \\
\hline TS-1 & 32.8 & 389.3 & 271.6 & 117.7 & 0.14 & 0.07 \\
HTS-1 & 34.0 & 413.9 & 272.0 & 141.9 & 0.14 & 0.20 \\
\hline
\end{tabular}

Note: ${ }^{\mathrm{a}} \mathrm{The}$ molar ratio of Si/Ti was measured by ICP-OES.

The TEM images of HTS-1 indicate that there are a large number of mesopores and macropores in the sample (Fig. 5a). As shown in Fig. 5b, HTS-1 has both intracrystalline and intercrystalline pores (oval area), and some of the mesopores or macropores are directly connected to the crystal surface. In addition, HTS1 presents a clear lattice line of the MFI topology (the diamond-shaped area), and its micropore size is about $0.5 \mathrm{~nm}$. The above results show that some ordered micropores of HTS- 1 crystal are connected with mesopores or macropores, and then an interconnected hierarchical pore system is formed.
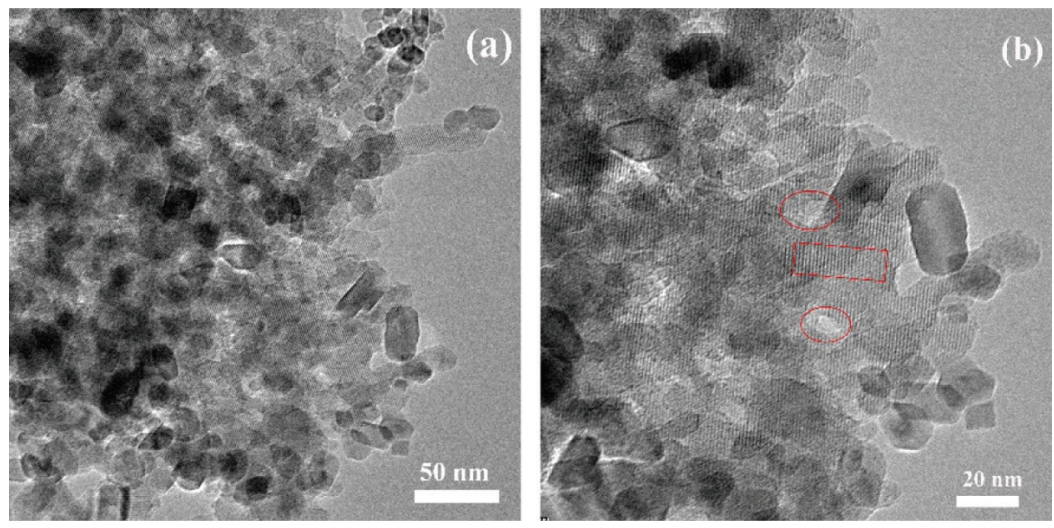

Figure 5: TEM images of HTS-1 taken at low (a) and high (b) magnification

\subsection{Catalytic Performance on Oxidative Desulfurization}

\subsubsection{Effect of Reaction Conditions}

Table 2 shows the effect of different reaction conditions on the performance of HTS-1 for the ODS of DBT. It can be seen that, with the increase of reaction temperature from 323 to $343 \mathrm{~K}$, the conversion of DBT first increases and then decreases slowly, which is due to the decomposition of TBHP at higher temperature. With the increase of TBHP/S molar ratio from 1 to 3, the conversion of DBT first increases suddenly and then increases slowly. Further increasing the TBHP/S molar ratio, the conversion of DBT changes little. As the catalyst to oil ratio increases from 3 to $5 \mathrm{~g} / \mathrm{L}$, the conversion of DBT increases. Further increasing the catalyst to oil ratio, the conversion of DBT keeps constant. With the reaction time prolongs from 2 to $3 \mathrm{~h}$, the conversion of DBT increases slightly. Further prolonging the reaction time, the conversion of DBT keeps constant. Therefore, the appropriate reaction conditions are as follows: temperature is $333 \mathrm{~K}$, molar ratio of TBHP/S is 3.0, catalyst to oil ratio is $5 \mathrm{~g} / \mathrm{L}$, and reaction time is $3 \mathrm{~h}$.

Table 3 shows the effect of different reaction conditions on the performance of HTS- 1 for the ODS of 4,6-DMDBT. As one can see from Table 3, with the increase of reaction temperature from $323 \mathrm{~K}$ to $343 \mathrm{~K}$, the conversion of 4,6-DMDBT first increases and then decreases slightly, which is due to the decomposition of TBHP at higher temperature. With the increase of TBHP/S molar ratio from 1 to 3 , the conversion of 4,6- 
DMDBT first increases suddenly and then increase slightly. Further increasing the TBHP/S molar ratio, the conversion of 4,6-DMDBT keeps constant. This is because the oxidation of one molecule of DBT or 4,6DMDBT to produce the corresponding sulfone requires two molecules of TBHP to be consumed [38]. Therefore, when the TBHP/S ratio increases from 1 to 2 , the conversion of DBT or 4,6-DMDBT increases rapidly. With the catalyst to oil ratio increases from 3 to $5 \mathrm{~g} / \mathrm{L}$, the conversion of 4,6-DMDBT increases. Further increasing the catalyst to oil ratio, the conversion of 4,6-DMDBT changes little. With the reaction time prolongs from 1 to $3 \mathrm{~h}$, the conversion of 4,6-DMDBT first increases slowly and then keeps constant. Thus, the optimum reaction conditions are as follows: temperature is $333 \mathrm{~K}$, molar ratio of TBHP/S is 3.0, catalyst to oil ratio is $5 \mathrm{~g} / \mathrm{L}$, and reaction time is $2 \mathrm{~h}$.

Table 2: Effect of reaction parameters on the ODS of DBT catalyzed by HTS-1

\begin{tabular}{llllll}
\hline Entry & Temp. $(\mathrm{K})$ & Cat./Oil $(\mathrm{g} / \mathrm{L})$ & TBHP/S molar ratio & Time $(\mathrm{h})$ & $X_{\text {DBT }}(\%)$ \\
\hline 1 & 323 & 5 & 3.0 & 3 & 96.1 \\
2 & 333 & 5 & 3.0 & 3 & 99.6 \\
3 & 343 & 5 & 3.0 & 3 & 99.1 \\
4 & 333 & 5 & 1.0 & 3 & 58.2 \\
5 & 333 & 5 & 2.0 & 3 & 98.5 \\
6 & 333 & 5 & 4.0 & 3 & 99.5 \\
7 & 333 & 5 & 6.0 & 3 & 99.7 \\
8 & 333 & 3 & 3.0 & 3 & 94.4 \\
9 & 333 & 4 & 3.0 & 3 & 98.1 \\
10 & 333 & 6 & 3.0 & 3 & 99.7 \\
11 & 333 & 7 & 3.0 & 3 & 99.8 \\
12 & 333 & 5 & 3.0 & 2 & 97.6 \\
13 & 333 & 5 & 3.0 & 4 & 99.7 \\
\hline
\end{tabular}

Table 3: Effect of reaction parameters on the ODS of 4,6-DMDBT catalyzed by HTS-1

\begin{tabular}{llllll}
\hline Entry & Temp. (K) & Cat./Oil $(\mathrm{g} / \mathrm{L})$ & TBHP/S molar ratio & Time $(\mathrm{h})$ & $X_{4,6-\text { DMDBT }}(\%)$ \\
\hline 1 & 323 & 5 & 3.0 & 2 & 97.0 \\
2 & 333 & 5 & 3.0 & 2 & 99.6 \\
3 & 343 & 5 & 3.0 & 2 & 99.4 \\
4 & 333 & 5 & 1.0 & 2 & 60.3 \\
5 & 333 & 5 & 2.0 & 2 & 98.8 \\
6 & 333 & 5 & 4.0 & 2 & 99.6 \\
7 & 333 & 5 & 6.0 & 2 & 99.7 \\
8 & 333 & 3 & 3.0 & 2 & 96.7 \\
9 & 333 & 4 & 3.0 & 2 & 98.3 \\
\hline
\end{tabular}


JRM, 2022, vol.10, no.6

1719

\begin{tabular}{llllll}
\hline \multicolumn{2}{c}{ Table 3 (continued). } & & & \\
\hline Entry & Temp. (K) & Cat./Oil (g/L) & TBHP/S molar ratio & Time (h) & $X_{4,6-\text { DMDBT }}(\%)$ \\
\hline 10 & 333 & 6 & 3.0 & 2 & 99.5 \\
11 & 333 & 7 & 3.0 & 2 & 99.9 \\
12 & 333 & 5 & 3.0 & 1 & 98.5 \\
13 & 333 & 5 & 3.0 & 3 & 99.7 \\
\hline
\end{tabular}

Under the above optimized reaction conditions, the influence of reaction time on the catalytic performances of TS-1 and HTS-1 was studied in detail (Fig. 6). For TS-1, the conversion of DBT after $3 \mathrm{~h}$ of reaction is $72.6 \%$, and the conversion of 4,6-DMDBT after $2 \mathrm{~h}$ of reaction is $83.1 \%$. However, the catalytic activity of HTS-1 is much higher than that of TS- 1 . The conversions of DBT and 4,6-DMDBT both exceed $99.5 \%$. Moreover, the catalyst blank experiments and oxidant blank experiments showed that the conversions of DBT and 4,6-DMDBT are both less than 5.0\% (data are not displayed). Because of the intrinsic micropores (about $0.55 \mathrm{~nm}$ ) of TS-1, bulky sulfur compounds such as DBT and 4,6-DMDBT do not diffuse into the porous channels to interact with the titanium active sites. Therefore, the lower catalytic activities of TS-1 in the ODS of DBT and 4,6-DMDBT are mainly attributed to titanium active sites on the outer surface of zeolite. In addition, due to the similar titanium content of TS-1 and HTS-1 (Table 1), the increase in HTS-1 catalytic activity should be due to the improved accessibility of active sites by DBT and 4,6-DMDBT [18]. Compared with TS-1, the $\mathrm{S}_{\mathrm{BET}}, \mathrm{V}_{\text {meso }}$ and $\mathrm{S}_{\text {ext }}$ of HTS-1 are much higher, which is beneficial for bulky reactants to approach active sites and overcome the limitation of diffusion (Table 1). Meanwhile, the HTS-1 crystal is an aggregate structure formed by the accumulation of small nanoparticles with a grain size of 15-140 nm, and it has a system of interconnected hierarchical pores (Figs. 5 and 7a). These interconnected mesoporous or macroporous pores facilitate the transport of bulky reactants and products, thereby shortening the diffusion path of reactants and products [21]. Therefore, HTS-1 exhibits excellent catalytic oxidation performances in the ODS of DBT and 4,6DMDBT.

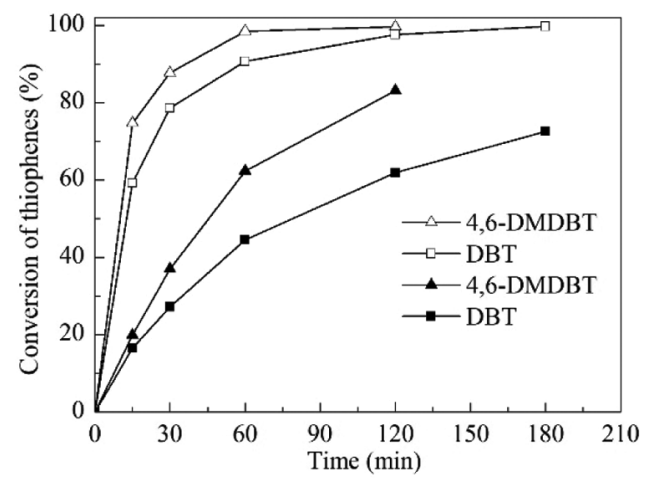

Figure 6: The ODS of DBT and 4,6-DMDBT over TS-1 $(\boldsymbol{\square}, \mathbf{\Delta})$ and HTS-1 $(\square, \triangle)$. Reaction conditions: model fuels, $10 \mathrm{~mL}$; catalyst to oil ratio, $5 \mathrm{~g} / \mathrm{L}$; TBHP/S molar ratio, 3.0; temperature, $333 \mathrm{~K}$ 

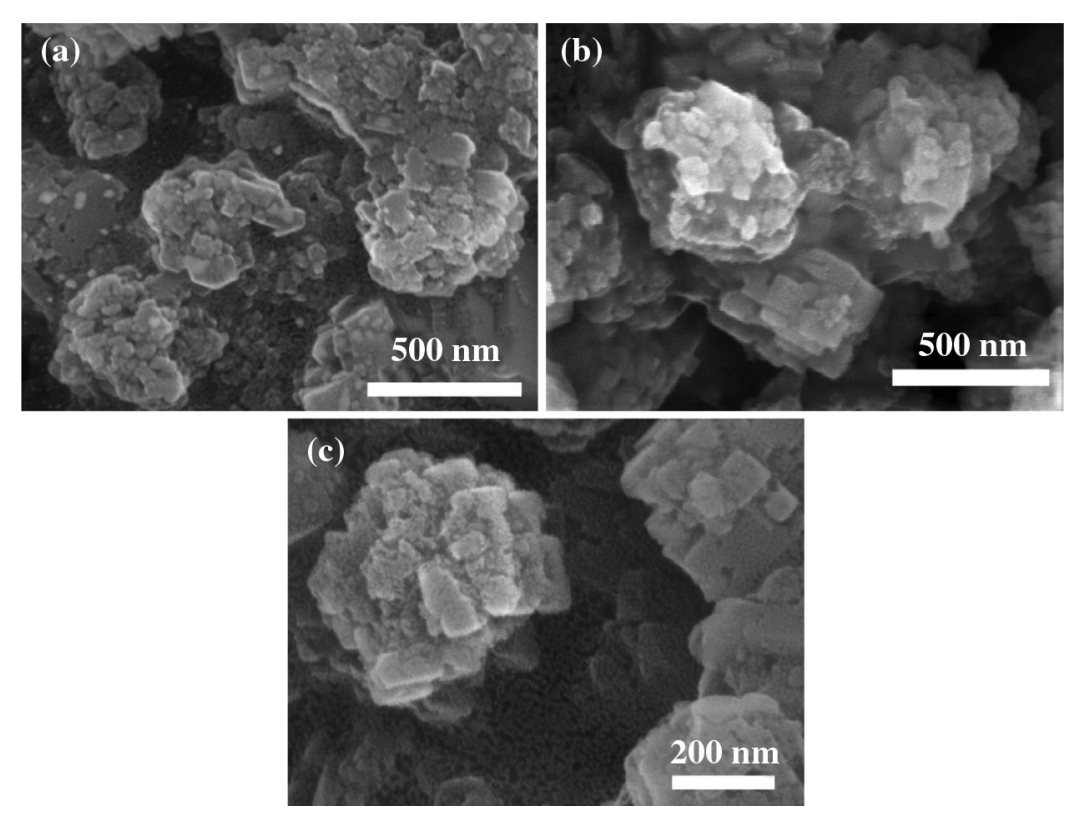

Figure 7: SEM images of the fresh HTS-1 (a), HTS-1cc15 regenerated from the ODS of 4,6-DMDBT (b), and HTS-1cc15 regenerated from the ODS of DBT (c)

\subsubsection{Recycling Performance of HTS-1}

Under the optimum reaction conditions, the recycle tests for the ODS of DBT and 4,6-DMDBT over HTS-1c samples are carried out, and the results are shown in Fig. 8. As the number of cyclic experiments increased from 1 to 5, the conversions of DBT and 4,6-DMDBT decrease linearly. It is worth noting that the conversion of 4,6 -DMDBT is $71.6 \%$ after the 5 th run.

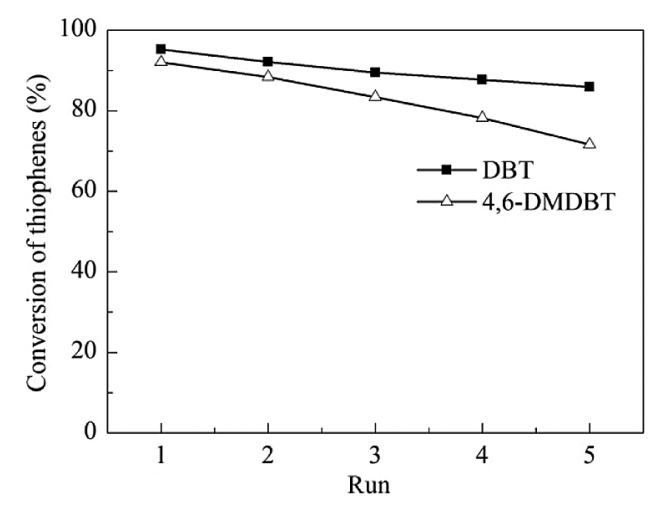

Figure 8: Recycle tests for the catalytic oxidation of DBT and 4,6-DMDBT over HTS-1c samples

In order to reveal the reasons for the decrease of the catalytic activity of HTS-1c, CHN analysis, FT-IR and UV-Vis techniques are used to characterize the oxidation products and HTS- $1 \mathrm{c} 5$ samples. The results of CHN analysis show that the carbon content of HTS- $1 \mathrm{c} 5$ recovered from the ODS of DBT is $6.63 \mathrm{wt} . \%$, and that of HTS-1c5 recovered from the ODS of 4,6-DMDBT is $41.19 \mathrm{wt} . \%$. It is worth noting that we have confirmed in a previous report [33] that DBT and 4,6-DMDBT are catalyzed by HTS-1 to generate corresponding sulfones. The HTS-1c5 samples all have an absorption peak at $2957 \mathrm{~cm}^{-1}$ (Figs. $3 \mathrm{c}$ and $3 \mathrm{~d}$ ), which is attributed to the stretching vibration of the $\mathrm{C}-\mathrm{H}$ bond [7,39], indicating that the HTS-1c5 samples contain some oxidation products [33]. Moreover, the HTS-1c5 sample recovered from 
the ODS of 4,6-DMDBT shows obvious absorption peaks at 1282, 1149 and $601 \mathrm{~cm}^{-1}$, which belong to asymmetric stretching vibration, symmetric stretching vibration and shear vibration of sulfones $(\mathrm{O}=\mathrm{S}=\mathrm{O})$, respectively [39]. The above results indicate that part of oxidation products of DBT and 4,6-DMDBT are adsorbed on the HTS-1c5 samples, which is consistent with the results of CHN analysis.

As shown in Fig. 9, the oxidation products of DBT and 4,6-DMDBT have a strong absorption band at about $230 \mathrm{~nm}$, and a broad shoulder band at 260-380 nm. Compared with fresh HTS-1, the absorption intensity of the main band of HTS- $1 \mathrm{c} 5$ samples decreases and redshifts to $215 \mathrm{~nm}$. In addition, HTS$1 \mathrm{c} 5$ used for DBT oxidation has a slight decrease in the absorption intensity of the band at about $330 \mathrm{~nm}$, while HTS-1c5 used for 4,6-DMDBT oxidation has a significant increase in the absorption intensity of the band at $290-380 \mathrm{~nm}$. These results and the FT-IR results described above demonstrate that the oxidation products of DBT and 4,6-DMDBT remain in the recovered samples. They, especially the oxidation product of 4,6-DMDBT, is easier to adsorb on the active sites or channels, which makes the conversion of 4,6-DMDBT decrease more obviously during the recycle tests. The above results indicate that samples recovered by centrifugation is prone to deactivation, and their recycling performances are poor.
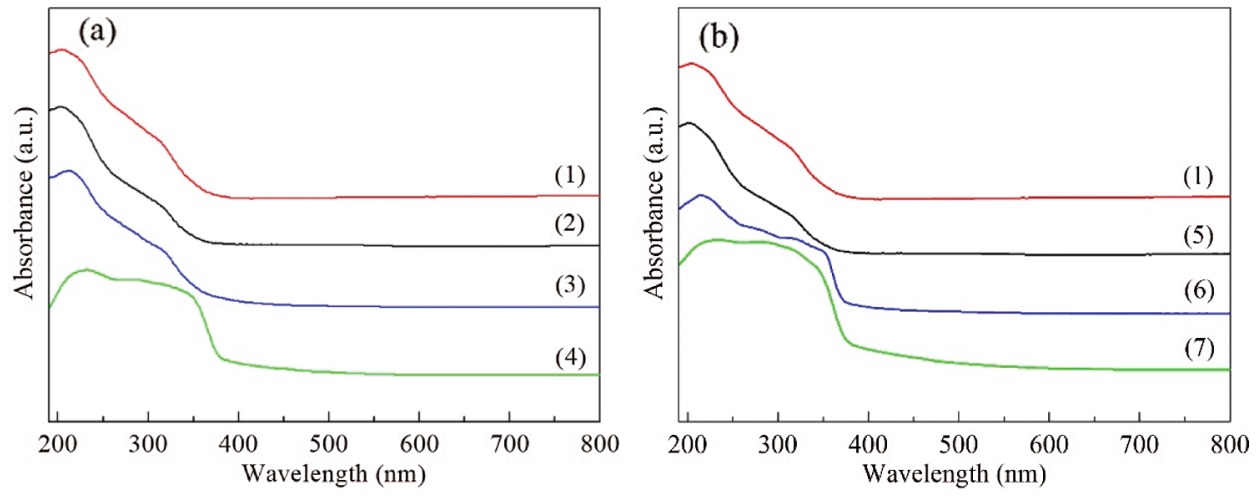

Figure 9: UV-Vis spectra of HTS-1, HTS-1c and HTS-1cc for the ODS of DBT (a) and 4,6-DMDBT (b): (1) fresh HTS-1; (2) HTS-1cc15 regenerated from the ODS of DBT; (3) HTS-1c5 recovered from the ODS of DBT; (4) oxidation product of DBT separated from the solid mixture after reaction; (5) HTS1 cc15 regenerated from the ODS of 4,6-DMDBT; (6) HTS-1c5 recovered from the ODS of 4,6-DMDBT; (7) oxidation product of 4,6-DMDBT separated from the solid mixture after reaction

Under the optimum reaction conditions, the recycle tests for the oxidation of DBT and 4,6-DMDBT over HTS-1cc samples are conducted, and the results are shown in Fig. 10. After the 15th run, HTS-1cc15 samples still maintain high conversions of DBT (90.6\%) and 4,6-DMDBT (86.0\%) without deactivation. The HTS$1 \mathrm{cc} 15$ samples were further characterized by XRD, UV-Vis and SEM techniques. As shown in Fig. 1, compared with fresh HTS-1, the regenerated samples still show typical diffraction peaks of MFI topology at $7.9^{\circ}, 8.8^{\circ}, 23.0^{\circ}, 23.9^{\circ}$ and $24.3^{\circ}$, and the intensity of these peaks does not change much, suggesting that the crystal structure of samples is well maintained. As shown in Fig. 9, the absorption curves of HTS-1cc15 samples recovered to be similar to that of the fresh sample, indicating that the adsorbed desulfurization products are effectively removed by calcinating. Compared with fresh HTS-1, the absorption intensity of band at $204 \mathrm{~nm}$ does not change much, indicating that the isolated $\mathrm{Ti}$ (IV) species in the framework of HTS-1cc15 samples remain intact. Fig. 7 shows the SEM images of HTS-1 and HTS-1cc15. One can see that the crystal morphology and size of HTS-1cc15 samples are similar to HTS1, indicating that their crystal morphology are well kept. The above results demonstrate that HTS-1 has good structural stability and recycling performance. As shown in Table 4, compared with the earlier ODS 
catalysts (formic acid and acetic acid), the HTS-1 in this work has better oxidative desulfurization performance. Moreover, compared with HTS-1 zeolites prepared by different methods reported in literature (Table 4) $[38,40,41]$, the recycling performance of HTS-1 prepared in this work is equivalent to or better than that of the reported samples.

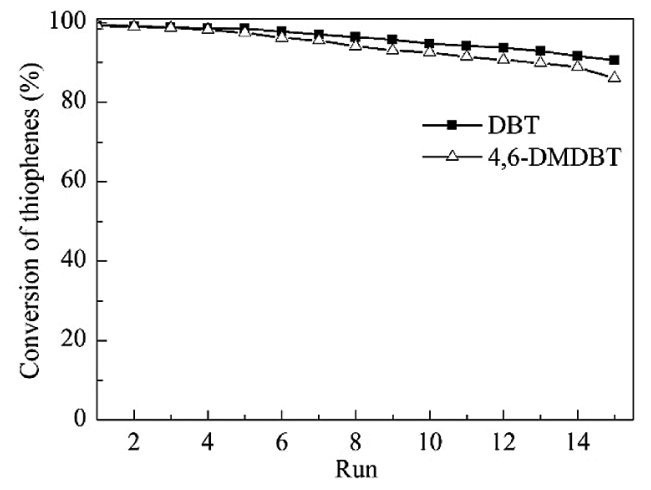

Figure 10: Recycle tests for the catalytic oxidation of DBT and 4,6-DMDBT over HTS-1cc samples

Table 4: Recycle tests for the catalytic oxidation of DBT and 4,6-DMDBT (or gas oils) over different catalysts

\begin{tabular}{lllllll}
\hline Catalyst & Ti/Si molar ratio & Temp. (K) & Cyclic number & $X_{\text {DBT }}{ }^{\mathrm{a}}(\%)$ & $X_{4,6-D M D B T}{ }^{\mathrm{a}}(\%)$ & Reference \\
\hline Formic acid & - & 323 & - & $92.6^{\mathrm{b}}$ & - & {$[7]$} \\
Acetic acid & - & 363 & - & $>90^{\mathrm{c}}$ & - & {$[10]$} \\
HTS-1B & 0.045 & 333 & 4 & $\sim 99$ & - & {$[17]$} \\
HTS-1-A & 0.033 & 333 & 3 & 99.0 & - & {$[18]$} \\
HTS-1 & 0.024 & 333 & 4 & $\sim 99$ & - & {$[10]$} \\
HTS-1b & 0.016 & 333 & 3 & 99 & 99 & {$[41]$} \\
TS-1(H3) & 0.012 & 333 & 7 & 92 & - & {$[38]$} \\
\hline
\end{tabular}

Note: ${ }^{\text {a }}$ The catalytic results of the last cyclic reaction of DBT and 4,6-DMDBT, respectively.

${ }^{\mathrm{b}}$ Overall desulfurization rate of straight run-light gas oil (SR-LGO, Sulfur content: $1.35 \mathrm{wt} \%$ ) after catalytic oxidation and acetonitrile extraction.

${ }^{\mathrm{c}}$ Overall desulfurization rate of straight-run gas oil (gas oil B, Sulfur content: $2.40 \mathrm{wt} \%$ ) after catalytic oxidation and methanol extraction.

\subsection{Reaction Mechanism of ODS Process}

It is generally believed that, for the catalytic system containing titanosilicate zeolites and hydroperoxide, the Ti-peroxo species produced by the reaction of titanium active sites with either organic hydroperoxides or hydrogen peroxide act as the active intermediate in the oxidation of various organic substrates [42-44]. In order to capture the Ti-peroxo species, the samples are treated with TBHP aqueous solution and detected by the UV-Vis technique. As shown in Fig. 2a, after TBHP aqueous solution treatment, a new absorption band at $370-550 \mathrm{~nm}$ for TS-1 and HTS-1 appears, which is attributed to the charge transfer transition from the peroxo ligand to the Ti center in the Ti-peroxo species, indicating the formation of Ti-peroxo $\left(\mathrm{Ti}-\mathrm{OO}^{t} \mathrm{Bu}\right)$ species [42-44]. In addition, the same treatments were performed on HTS-1cc15 samples (Fig. 2b), and they all show an obvious absorption band at 370-550 nm, indicating that the regenerated samples still have a strong ability to combine TBHP to form Ti-OO ${ }^{t} \mathrm{Bu}$ species after the 15 th run. The results are consistent with the results of recycle tests of HTS-1cc samples.

Based on the above results, the ODS reaction mechanism of DBT and 4,6-DMDBT on HTS-1 with TBHP is proposed (Scheme 1). The reaction pathway begins with the formation of the active intermediate 
Ti-OO ${ }^{t} \mathrm{Bu}$ species, which is generated by the reaction of TBHP and titanium active sites. The oxidation process is carried out by nucleophilic attack of sulfur atoms in the DBT (or 4,6-DMDBT) on the Ti$\mathrm{OO}^{t} \mathrm{Bu}$ species to form sulfoxides and regenerated HTS-1. The sulfoxides are very unstable and quickly oxidized by the Ti-OO ${ }^{t} \mathrm{Bu}$ species into sulfones [33]. Moreover, since sulfones are insoluble in n-octane, the products and catalysts can be precipitated from the reaction medium immediately after the reaction, which helps to remove them from model fuels by simple centrifugation or filtration [32].

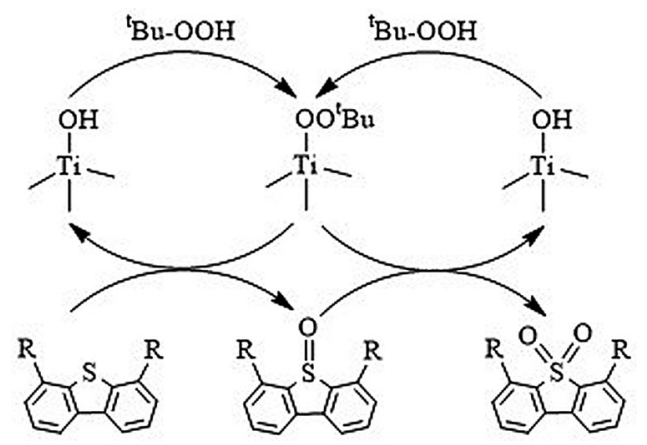

Scheme 1: A proposed cyclic mechanism for the ODS of DBT and 4,6-DMDBT by TBHP in the presence of HTS-1 catalyst ( $\mathrm{R}$ represents $\mathrm{H}$ or $\mathrm{CH}_{3}$ ).

\section{Conclusions}

In summary, HTS- 1 was prepared using $\mathrm{C}-\mathrm{SiO}_{2}$ composite as a mesoporous template and TPAOH as a microporous template. Compared with TS-1, the $\mathrm{V}_{\text {micro }}$ of HTS- 1 is similar, but the $\mathrm{S}_{\mathrm{BET}}, \mathrm{S}_{\mathrm{ext}}$ and $\mathrm{V}_{\text {meso }}$ of HTS-1 are significantly increased. Therefore, HTS-1 exhibits higher catalytic activities in the ODS of DBT and 4,6-DMDBT. The effects of different reaction parameters on the performances of HTS-1 for the ODS of DBT and 4,6-DMDBT are investigated, and the optimum reaction conditions are: temperature is $333 \mathrm{~K}$, molar ratio of TBHP/S is 3.0, catalyst to oil ratio is $5 \mathrm{~g} / \mathrm{L}$, and reaction time of DBT and 4,6-DMDBT are 3 and $2 \mathrm{~h}$, respectively. Under optimum reaction conditions, the recycling performances of HTS- 1 for the ODS of DBT and 4,6-DMDBT are studied. The results show that the catalytic activity of HTS-1c recovered by centrifugation decreases linearly with the increase of the number of cyclic experiments, which is due to the adsorption or aggregation of oxidation products on the active sites or channels. When the samples were regenerated by calcination at $823 \mathrm{~K}$, their catalytic activities are restored. After the 15th run, HTS-1cc samples still keep high conversions of DBT (90.6\%) and 4,6-DMDBT (86.0\%) without deactivation. The results of related characterizations show that the crystal structure and morphology of samples are maintained well after cyclic experiments. By treating the samples with TBHP aqueous solution, the $\mathrm{Ti}^{-} \mathrm{OO}^{t} \mathrm{Bu}$ species, which are the active intermediates of oxidative desulfurization, are captured through the UV-Vis technique. Moreover, a possible mechanism of oxidative desulfurization for bulky sulfides over HTS-1 is proposed. This strategy can be applied to prepare a series of heteroatom-substituted MFI zeolites with hierarchical channels.

Acknowledgement: This work is supported by the National Natural Science Foundation of China (21303008) and Natural Science Foundation of Hubei Province of China (2012FFB00103).

Funding Statement: This research was funded by the National Natural Science Foundation of China (21303008) and Natural Science Foundation of Hubei Province of China (2012FFB00103).

Conflicts of Interest: The authors declare that they have no conflicts of interest to report regarding the present study. 


\section{References}

1. Rajendran, A., Cui, T. Y., Fan, H. X., Yang, Z. F., Feng, J. et al. (2020). A comprehensive review on oxidative desulfurization catalysts targeting clean energy and environment. Journal of Materials Chemistry A, 8(5), 2246-2285. DOI 10.1039/C9TA12555H.

2. Hao, L. D., Hurlock, M. J., Li, X. Y., Ding, G. D., Kriegsman, K. W. et al. (2020). Efficient oxidative desulfurization using a mesoporous Zr-based MOF. Catalysis Today, 350, 64-70. DOI 10.1016/j. cattod.2019.04.012.

3. Srivastava, V. C. (2012). An evaluation of desulfurization technologies for sulfur removal from liquid fuels. $R C S$ Advances, 2(3), 759-783. DOI 10.1039/C1RA00309G.

4. Kwon, J. M., Moon, J. L., Bae, Y. S., Lee, D. G., Sohn, H. C. et al. (2008). Adsorptive desulfurization and denitrogenation of refinery fuels using mesoporous silica adsorbents. Chemsuschem, 1(4), 307-309. DOI 10.1002/(ISSN)1864-564X.

5. Abro, R., Abdeltawab, A. A., Al-Deyab, S. S., Yu, G. R., Qazi, S. R. et al. (2014). A review of extractive desulfurization of fuel oils using ionic liquids. Rsc Advances, 4(67), 35302-35317. DOI 10.1039/C4RA03478C.

6. Monticello, D. J. (2000). Biodesulfurization and the upgrading of petroleum distillates. Current Opinion in Biotechnology, 11(6), 540-546. DOI 10.1016/S0958-1669(00)00154-3.

7. Otsuki, S., Nonaka, T., Takashima, N., Qian, W. H., Ishihara, A. et al. (2000). Oxidative desulfurization of light Gas Oil and vacuum Gas Oil by oxidation and solvent extraction. Energy \& Fuels, 14(6), 1232-1239. DOI 10.1021/ef000096i.

8. Murata, S., Murata, K., Kidena, K., Nomura, M. (2004). A novel oxidative desulfurization system for diesel fuels with molecular oxygen in the presence of cobalt catalysts and aldehydes. Energy \& Fuels, 18(1), 116-121. DOI 10.1021/ef034001z.

9. Yazu, K., Makino, M., Ukegawa, K. (2004). Oxidative desulfurization of diesel oil with hydrogen peroxide in the presence of acid catalyst in diesel oil/acetic acid biphasic system. Chemistry Letters, 33(10), 1306-1307. DOI 10.1246/cl.2004.1306.

10. Zannikos, F., Lois, E., Stournas, S. (1995). Desulfurization of petroleum fractions by oxidation and solvent extraction. Fuel Processing Technology, 42(1), 35-45. DOI 10.1016/0378-3820(94)00104-2.

11. Du, Y., Zhou, L. N., Liu, Z. H., Yang, L. (2021). Polyoxometalate-based 3DOM ZrO (2) material for deep oxidative desulfurization of DBT with ultrahigh stability. Journal of Porous Materials, 28(1), 109-116. DOI 10.1007/s10934-020-00969-y.

12. Yan, X. M., Mei, Z. K., Mei, P., Yang, Q. F. (2014). Self-assembled HPW/silica-alumina mesoporous nanocomposite as catalysts for oxidative desulfurization of fuel oil. Journal of Porous Materials, 21(5), 729737. DOI 10.1007/s10934-014-9819-2.

13. Li, S. W., Wang, W., Zhao, J. S. (2020). Highly effective oxidative desulfurization with magnetic MOF supported W-moO3 catalyst under oxygen as oxidant. Applied Catalysis B: Environmental, 277, 119224. DOI 10.1016/j. apcatb.2020.119224.

14. Hulea, V., Fajula, F. C., Bousquet, J. (2001). Mild oxidation with $\mathrm{H}_{2} \mathrm{O}_{2}$ over Ti-containing molecular sieves-a very efficient method for removing aromatic sulfur compounds from fuels. Journal of Catalysis, 198(2), 179-186. DOI 10.1006/jcat.2000.3149.

15. Wang, W. H., Li, G., Li, W. G., Liu, L. P. (2011). Synthesis of hierarchical TS-1 by caramel templating. Chemical Communications, 47(12), 3529-3531. DOI 10.1039/c0cc04560h.

16. Jin, C. Z., Li, G., Wang, X. S., Zhao, L. X., Liu, L. P. et al. (2007). Synthesis, characterization and catalytic performance of Ti-containing mesoporous molecular sieves assembled from titanosilicate precursors. Chemistry of Materials, 19(7), 1664-1670. DOI 10.1021/cm0625777.

17. Du, S. T., Chen, X. X., Sun, Q. M., Wang, N., Jia, M. J. et al. (2016). A non-chemically selective top-down approach towards the preparation of hierarchical TS-1 zeolites with improved oxidative desulfurization catalytic performance. Chemical Communications, 52(17), 3580-3583. DOI 10.1039/C5CC10232D. 
18. Du, S. T., Li, F., Sun, Q. M., Wang, N., Jia, M. J. et al. (2016). A green surfactant-assisted synthesis of hierarchical TS-1 zeolites with excellent catalytic properties for oxidative desulfurization. Chemical Communications, 52(16), 3368-3371. DOI 10.1039/C5CC08441E.

19. Du, S. T., Sun, Q. M., Wang, N., Chen, X. X., Jia, M. J. et al. (2017). Synthesis of hierarchical TS-1 zeolites with abundant and uniform intracrystalline mesopores and their highly efficient catalytic performance for oxidation desulfurization. Journal of Materials Chemistry A, 5(17), 7992-7998. DOI 10.1039/C6TA10044A.

20. Wang, X. X., Li, G., Wang, W. H., Jin, C. Z., Chen, Y. Y. (2011). Synthesis, characterization and catalytic performance of hierarchical TS-1 with carbon template from sucrose carbonization. Microporous and Mesoporous Materials, 142(2-3), 494-502. DOI 10.1016/j.micromeso.2010.12.035.

21. Fang, Y. M., Hu, H. Q. (2007). Mesoporous TS-1: Nanocasting synthesis with CMK-3 as template and its performance in catalytic oxidation of aromatic thiophene. Catalysis Communications, 8, 817-820. DOI 10.1016/j.catcom.2006.09.018.

22. Tanev, P. T., Chlbwe, M., Plnnavala, T. J. (1994). Titanium-containing mesoporous molecular sieves for catalytic oxidation of aromatic compounds. Nature, 368(6469), 321-323. DOI 10.1038/368321a0.

23. Koyano, K. A., Tatsumi, T. (1996). Synthesis of titanium-containing mesoporous molecular sieves with a cubic structure. Chemical Communications, 32(2), 145-146. DOI 10.1039/cc9960000145.

24. Wilde, N., Pelz, M., Gebhardt, S. G., Gläser, R. (2015). Highly efficient nano-sized TS-1 with micro-/mesoporosity from desilication and recrystallization for the epoxidation of biodiesel with $\mathrm{H}_{2} \mathrm{O}_{2}$. Green Chemistry, 17(6), 33783389. DOI 10.1039/C5GC00406C.

25. Wang, B. R., Lin, M., Peng, X. X., Zhu, B., Shu, X. T. (2016). Hierarchical TS-1 synthesized effectively by postmodification with TPAOH and ammonium hydroxide. RSC Advances, 6(51), 44963-44971. DOI 10.1039/ C6RA06657G.

26. Wang, B. R., Peng, X. X., Zhang, W. F., Lin, M., Zhu, B. et al. (2017). Hierarchical TS-1 synthesized via the dissolution-recrystallization process influence of ammonium salts. Catalysis Communications, 101, 26-30. DOI 10.1016/j.catcom.2017.07.016.

27. Wang, B. R., Lin, M., Zhu, B., Peng, X. X., Xu, G. T. et al. (2016). The synthesis, characterization and catalytic activity of the hierarchical TS-1 with the intracrystalline voids and grooves. Catalysis Communications, 75, 69-73. DOI 10.1016/j.catcom.2015.12.007.

28. Wei, Y., Li, G., Lü, Q., Cheng, C. Y., Cuo, H. C. et al. (2018). Green and efficient epoxidation of methyl oleate over hierarchical TS-1. Chinese Journal of Catalysis, 39(5), 964-972. DOI 10.1016/S1872-2067(18)63014-1.

29. Cheneviere, Y., Chieux, F., Caps, V., Tuel, A. (2010). Synthesis and catalytic properties of TS-1 with mesoporous/ microporous hierarchical structures obtained in the presence of amphiphilic organosilanes. Journal of Catalysis, 269(1), 161-168. DOI 10.1016/j.jcat.2009.11.003.

30. Schmidt, I., Boisen, A., Gustavsson, E., Ståhl, K., Pehrson, S. et al. (2001). Carbon nanotube templated growth of mesoporous zeolite single crystals. Chemistry of Materials, 13(12), 4416-4418. DOI 10.1021/cm011206h.

31. Du, Q., Guo, Y. P., Duan, H. A., Li, H., Chen, Y. J. et al. (2017). Facile preparation of hierarchical titanium silicalite-1 (TS-1) with efficient oxidation of cyclic alkenes using PVA modified MWCNTs as templates. Journal of Alloys and Compounds, 699, 386-391. DOI 10.1016/j.jallcom.2016.12.382.

32. Pei, X. X., Liu, X. X., Liu, X. Y., Shan, J. L., Fu, H. et al. (2019). Synthesis of hierarchical titanium silicalite1 using a carbon-silica-titania composite from xerogel mild carbonization. Catalysts, 9(8), 672. DOI 10.3390/ catal9080672.

33. Shan, J. L., Liu, X. X., Huai, J. L., Zhang, Y. H., Liu, X. Y. et al. (2021). Synthesis and characterization of hierarchical titanium silicalite-1 and its catalytic performance in oxidative desulfurization. Chinese Journal of Synthetic Chemistry, 29(6), 496-502. DOI 10.15952/j.cnki.cjsc.1005-1511.21005.

34. Wang, Z. D., Xu, L., Jiang, J. G., Liu, Y. M., He, M. Y. et al. (2012). One-pot synthesis of catalytically active and mechanically robust mesoporous TS-1 microspheres with the aid of triblock copolymer. Microporous and Mesoporous Materials, 156, 106-114. DOI 10.1016/j.micromeso.2012.02.025. 
35. Li, H., Lei, Q., Zhang, X. M., Suo, J. S. (2012). Nitrogen-incorporated TS-1 zeolite: Synthesis, characterization and application in the epoxidation of propylene. Microporous and Mesoporous Materials, 147(1), 110-116. DOI 10.1016/j.micromeso.2011.05.035.

36. Ricchiardi, G., Damin, A., Bordiga, S., Lamberti, C., Spanò, G. et al. (2001). Vibrational structure of titanium silicate catalysts. a spectroscopic and theoretical study. Journal of the American Chemical Society, 123(46), 11409-11419. DOI 10.1021/ja010607v.

37. Coudurier, G., Naccache, C., Vedrine, J. C. (1982). Uses of i.r. spectroscopy in identifying ZSM zeolite structure. Journal of the Chemical Society Chemical Communications, 18(24), 1413-1415. DOI 10.1039/C39820001413.

38. Bai, R. S., Sun, Q. M., Song, Y., Wang, N., Zhang, T. J. et al. (2018). Intermediate-crystallization promoted catalytic activity of titanosilicate zeolites. Journal of Materials Chemistry A, 6(18), 8757-8762. DOI 10.1039/ C8TA01960F.

39. Socrates, G. (2001). Infrared and raman characteristic group frequencies: Tables and charts. UK: Wiley Press.

40. Lv, G. J., Deng, S. L., Zhai, Y., Zhu, Y. Q., Li, H. C. et al. (2018). P123 lamellar micelle-assisted construction of hierarchical TS-1 stacked nanoplates with constrained mesopores for enhanced oxidative desulfurization. Applied Catalysis A: General, 567, 28-35. DOI 10.1016/j.apcata.2018.09.009.

41. Du, Q., Guo, Y. P., Wu, P., Liu, H. Z. (2018). Synthesis of hierarchically porous TS-1 zeolite with excellent deep desulfurization performance under mild conditions. Microporous and Mesoporous Materials, 264, 272-280. DOI 10.1016/j.micromeso.2018.01.015.

42. Bonino, F., Damin, A., Ricchiardi, G., Ricci, M., Spanò, G. et al. (2004). Ti-peroxo species in the TS-1/ $\mathrm{H}_{2} \mathrm{O}_{2} / \mathrm{H}_{2} \mathrm{O}$ system. Journal of Physical Chemistry B, 108(11), 3573-3583. DOI 10.1021/jp036166e.

43. Martausová, I., Spustová, D., Cvejn, D., Martaus, A., Lacný, Z. et al. (2019). Catalytic activity of advanced titanosilicate zeolites in hydrogen peroxide S-oxidation of methyl(phenyl)sulfide. Catalysis Today, 324, 144153. DOI 10.1016/j.cattod.2018.07.003.

44. Bordiga, S., Damin, A., Bonino, F., Ricchiardi, G., Lamberti, C. et al. (2002). The structure of the peroxo species in the TS-1 catalyst as investigated by resonant Raman spectroscopy. Angewandte Chemie International Edition, 41(24), 4734-4737. DOI 10.1002/(ISSN)1521-3773. 